\title{
Diacronie
}

Studi di Storia Contemporanea

$N^{\circ} 11,3$ | 2012

La satira fa storia. Eventi, pratiche, linguaggi

\section{Le vespe qualunquiste e la satira politica}

\section{Maurizio Cocco}

\section{(2) OpenEdition \\ Journals}

\section{Edizione digitale}

URL: http://journals.openedition.org/diacronie/2646

DOI: 10.4000/diacronie. 2646

ISSN: 2038-0925

\section{Editore}

Association culturelle Diacronie

\section{Notizia bibliografica digitale}

Maurizio Cocco, «Le vespe qualunquiste e la satira politica », Diacronie [Online], № 11, 3 | 2012,

documento 4, Messo online il 29 octobre 2012, consultato il 30 avril 2019. URL : http://

journals.openedition.org/diacronie/2646 ; DOI : 10.4000/diacronie.2646 


\title{
Diacronie
}

\section{Le vespe qualunquiste e la satira politica}

\author{
Maurizio COCCO *
}

Il più popolare giornale dell'Italia del secondo dopoguerra fu il settimanale romano «'Uomo Qualunque», ideato dal commediografo Guglielmo Giannini. Voce del malcontento e del disimpegno politico, l'«Uomo Qualunque» si opponeva alla nascente partitocrazia e ai governi de CLN utilizzando la satira come sua principale arma politica.

L'articolo analizza in modo particolare la rubrica «le vespe» nella quale Giannini, attraverso un uso smodato dello sberleffo, attaccava il nascente sistema e $i$ suoi uomini politici, criticava l'epurazione e metteva in ridicolo, con aneddoti e battute, quelli che giudicava gli aspetti più grotteschi della transizione dal fascismo alla vita democratica.

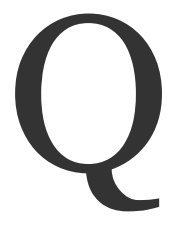
uesto non è un giornale umoristico, pur pubblicando caricature e vignette; non è un giornale "pesante", pur volendo onorarsi della collaborazione di grandi scrittori su argomenti di drammatico interesse; non è un giornale frivolo, pur non rinunziando alle pettegole Vespe. È il giornale dell'Uomo Qualunque, stufo di tutti, il cui solo ardente desiderio è che nessuno gli rompa più le scatole ${ }^{1}$.

Così si presentava, il 27 dicembre 1944, «L’Uomo Qualunque», un settimanale romano ideato e in larga parte scritto dal commediografo napoletano Guglielmo Giannini $^{2}$, destinato a essere - con una tiratura superiore alle 850.000 copie - il periodico di maggiore diffusione nell'Italia del secondo dopoguerra. Il successo fu tale

\footnotetext{
${ }^{1}$ GIANNINI, Guglielmo, in L'Uomo Qualunque (UQ), I, 1, 27 dicembre 1944.

2 Per un quadro generale su Guglielmo Giannini cfr. GIANNINI, Guglielmo, «La grande avventura dell'Uomo Qualunque raccontata da Guglielmo Giannini», in SCOGNAMIGLIO, Gennaro (a cura di), Enciclopedia del Centenario: Contributo alla storia politica, economica, letteraria e artistica dell'Italia meridionale nei primi cento anni di vita nazionale, vol. II, Napoli, D’Agostino, 1960.
} 
che il giornale diede vita a un partito politico (il Fronte dell'Uomo Qualunque) capace di conquistare trenta ${ }^{3}$ seggi all'Assemblea Costituente e affermarsi come partito di maggioranza in molti centri del Meridione in occasione delle elezioni amministrative dell'autunno 1946. Forte di un linguaggio semplice e diretto e di tematiche care alle necessità più immediate dell'uomo della strada, «L’Uomo Qualunque» fece della satira una delle armi più taglienti per denunciare il malcostume politico e attaccare la classe dirigente antifascista. Il giornale si serviva tanto della penna di Giannini, quanto dei disegni di Livio Apolloni, già collaboratore del «Travaso» e del «Marc'Aurelio» e di Giuseppe Russo, in arte Girus, collaboratore anche lui del «Travaso», ma anche dell'«Asino», del «Becco Giallo», del «Monocolo» e del «Corriere dei Piccoli». Non a caso il giornale si definiva un «settimanale politico satirico»4. Fin dal primo numero, Giannini utilizzò la caricatura e lo sberleffo per attaccare tutto ciò che non sopportava dell'Italia antifascista. Quest'uso della satira, per quanto presente in tutte le sezioni del giornale, trovò la sua più perfetta applicazione nella rubrica «Le Vespe», una serie di capoversi, spesso indipendenti l'uno dall'altro, così chiamati per la loro capacità di pungere e «accennare di volo»5, presentati come un efficace strumento di critica perché «a volte, sfottendo sfottendo...» si mettevano in risalto i veri problemi del Paese ${ }^{6}$. «Le Vespe» riprendevano l'omonima rubrica presente nel primo giornale che Giannini aveva fondato, a diciotto anni, «Il domani», ma vi si poteva avvertire l'affinamento guadagnato dallo scrittore attraverso la collaborazione, qualche anno dopo, al giornale umoristico napoletano «Monsignor Perelli». Presentate come una «rubrica di pettegolezzi» e basate su aneddoti curiosi e sul gusto per il ridicolo, trattavano comunque temi di forte impatto politico, al punto che Giannini sosteneva che questa fosse «la più importante rubrica politica d'Italia»7. Giannini amava andare controcorrente, si definiva un anarchico e, da buon uomo di teatro ${ }^{8}$, aveva il gusto per tutto ciò che causava stupore o che urtava la moralità altrui e proprio per questa ragione l'uso delle parolacce divenne, fin dal primo numero, uno dei caratteri distintivi del settimanale: «noi non le abbiamo mai vendute in borsa nera le nostre parolacce: le abbiamo regalate, prodigate, con inesausta generosità» 9 . Il linguaggio in sé - forse più del contenuto - fungeva da strumento di critica e di opposizione. Giannini voleva

\footnotetext{
3 Il gruppo qualunquista alla Costituente accolse poi altri sette deputati, eletti in altre liste.

4 GIANNINI, Guglielmo, «Le Vespe», in UQ, IV, 2, 8 gennaio 1947.

5 ID., «Le Vespe», in $U Q$, II, 2, 10 gennaio 1945.

6 ID., «Le Vespe», in $U Q$, II, 5, 31 gennaio 1945.

7 ID., «Le Vespe», in UQ, II, 15, 30 maggio 1945.

8 Giannini fu un commediografo di discreto successo, a lui può essere attribuita l'introduzione della commedia di genere poliziesco in Italia.

9 GIANNINI, Guglielmo, «Le Vespe», in UQ, III, 39, 25 settembre 1946.
} 
ostentatamente contrapporsi alla struttura di potere che si andava creando in Italia con la caduta del fascismo e rifiutava di riconoscerne la legittimità.

Allo stesso tempo la sua battaglia si estendeva alla politica come categoria. La sua opposizione era totale e partiva, appunto, da un uso della lingua che si poneva agli antipodi rispetto al linguaggio solitamente misurato dei politici. Per questa ragione, il principale bersaglio polemico della satira qualunquista furono i cosiddetti "uomini politici di professione", vale a dire i politici in generale, o i "Capi", persone giudicate incapaci di esercitare una professione e perciò dedite attraverso la politica a curare $\mathrm{i}$ propri interessi e accumulare fortune a danno dell'"uomo qualunque", della "Folla" in altre parole ${ }^{10}$. Già la scelta del nome era indicativa: rappresentava, "più che l'uomo della strada», «l'uomo nel caffè, nel cinematografo, nella camera da letto, nella sala da pranzo, davanti allo sportello delle tasse: dovunque», ma soprattutto «è un personaggio che si contrappone all'eroe, al capo, al re, al duce, al führer, al conducator, al Churchill, al Roosevelt, allo Stalin ${ }^{11}$. Segno distintivo dell'uomo politico di professione era il suo costante uso di lunghi discorsi per la maggior parte incomprensibili. «I discorsi son gli stessi, vuoti, piatti e monocordi: o ci scambiano per sordi, o ci pigliano per fessi» ${ }^{12}$. La contrapposizione fra "uomo politico" e "uomo qualunque", partiva quindi dal linguaggio per poi generare una visione della società assolutamente manichea. «Ma perché, perché la gente, crede sempre a chi più strilla, e per vivere tranquilla, lo fa duce, presidente, lo fa dèspota e padrone per averne calma e pace: e poi soffre, paga e tace, governata dal bastone?» ${ }^{13}$.

«L'Uomo Qualunque» non fu mai un giornale puramente satirico pur conservando un tono leggero e canzonatorio. Soprattutto nella fase d'istituzionalizzazione del partito, il giornale fu utilizzato anche come mezzo di diffusione di statuti, regolamenti e comunicazioni a militanti e simpatizzanti. La ripetitività era uno dei suoi aspetti peculiari: uno stesso tema veniva affrontato più volte e in modi diversi, ma è interessante notare come tutti i cardini del discorso qualunquista venissero spesso promossi attraverso aneddoti o barzellette. Ad esempio, il messaggio dell'antipolitica, ovvero del fatto che la politica fosse dannosa per il cittadino, veniva riassunto in questa storiella:

\footnotetext{
${ }^{10}$ Sulla distinzione manichea fra Capi e Folla cfr. GIANNINI, Guglielmo, La Folla: Seimila anni di lotta contro la tirannide, Soveria Mannelli, Rubbettino, 2002.

${ }^{11}$ GIANNINI, Guglielmo, La grande avventura dell'Uomo Qualunque raccontata da Guglielmo Giannini, cit., p. 41.

${ }_{12}$ ID., «Le Vespe», in $U Q$, II, 2, 10 gennaio 1945.

13 ID., «Le Vespe», in UQ, II, 12, 9 maggio 1945.
} 
Certo è che in una sera di ottobre, nella sala da pranzo dell'Hotel Storchen di Zurigo, uno scrittore italiano ha chiesto a suoi Amici, tutti residenti in Svizzera, come si chiamava il Capo dello Stato Svizzero: e nessuno ha saputo dirglielo. Profondamente impressionato da così superba indifferenza lo scrittore ha tentato la controprova, spingendo le sue indagini verso la cognizione del nome e cognome del ministro dell'interno, quindi di quello del tesoro. Non è riuscito a saperlo. Nella Svizzera, paese felice, la gente non sa come si chiamano coloro che governano. Lo Stato è anonimo. Tutto vi funziona perfettamente e ciò che conta è l'ufficio, non le generalità o il colore politico di chi lo occupa; la funzione, non il funzionario. Da noi, invece, mezza sinistra è favorevole al sottosegretario Corsi e lo esalta, l'altra mezza è contraria e lo impicca in effigie ${ }^{14}$.

La satira fu dunque, soprattutto nei primi passi di affermazione del giornale, il principale modello comunicativo qualunquista. La contestazione del sistema, e in particolare dei governi del CLN e delle leggi in materia di epurazione, si concretizzava nella messa in ridicolo, secondo il principio per il quale nella satira la pars destruens supera di gran lunga la pars costruens ${ }^{15}$. L'acronimo CLN veniva sciolto in «Consorzio Lavativi Nequitosi»16 e il "vento del Nord” trasformato in "rutto del Nord”. L'epurazione veniva invece rappresentata come un modo sporco di risolvere beghe personali, inaccettabile nei suoi scopi, perché pretendeva di mettere sotto processo un intero popolo che aveva solo pensato a "tirare a campare". «Se dall’impiego, benché capace, la commissione ti scaccerà, perché indossasti stivali e orbace, per familiari necessità, non ti stupisca tal decisione: è una fregnaccia d'epurazione» ${ }^{17}$. Si sosteneva che per l'impossibilità di colpire i cosiddetti pesci grossi si finisse con il colpire esclusivamente i pesci piccoli.

il povero questurino che ha arrestato il ladro inscritto al partito di sinistra è colpevole; Gronchi, sottosegretario con Mussolini, Casati, ministro con Mussolini, Sturzo, d'accordo con Mussolini, Croce, che vota per Mussolini, Orlando, che telegrafa a Mussolini, Nenni, che fonda i fasci per ordine di Mussolini, il gruppo comunista che guidato da Bombacci collabora con Mussolini, i liberali e i radicali che fiancheggiano Mussolini, oggi sono antifascisti; il disgraziato funzionarietto, che per guadagnare qualche soldarello di più ha preso la tessera, è fascista ${ }^{18}$.

\footnotetext{
14 ID., «Le Vespe», in UQ, II, 44, 30 ottobre 1946.

15 BRILLI, Attilio, Introduzione, in BRILLI, Attilio (a cura di), La Satira: Storia, tecniche, ideologie, Bari, Dedalo, 1979, p. 44.

16 GIANNINI, Guglielmo, «Le Vespe», in UQ, II, 25, 8 agosto 1945.

17 ID., «Le Vespe», in UQ, II, 28, 29 agosto 1945.

18 ID., «Le Vespe», in UQ, II, 27, 3 luglio 1946.
} 
L'espediente più usato per contestare il processo di epurazione era quello di pubblicare piccoli episodi di vita quotidiana, veri o verosimili, volti a metterne in risalto gli aspetti più grotteschi e contraddittori.

Nel regime di scompiglio per il padre paga il figlio, del colpevole presunto agli eredi è fatto appunto. Senonchè con questo andazzo sarà grave l'imbarazzo: il bastardo $\mathrm{e}$ il trovatello sarà il solo a farsi bello di sua stirpe ignota e sana di gran figlio di puttana ${ }^{19}$.

I partiti dell'antifascismo venivano descritti come i degni eredi del partito unico, vale a dire una cricca di politici di professione interessata solo ai propri affari. L'antifascismo diventava un fascismo sotto altro nome, continuatore della politica di discriminazione e oppressione che aveva afflitto l'Italia durante il ventennio. «Il fascismo è quel sistema che ciascuno vuol distruggere: per poter poi, senza tema, come lui sfruttare e suggere. È la vecchia, eterna storia, che ci stufa la cicoria: chi sta in basso ha fame e botte, chi sta in alto se ne fotte» ${ }^{20}$. Giannini denunciava il «fascismo dell'antifascismo», il proseguimento del dominio di un'oligarchia ai danni della maggioranza.

Ma la satira fu per Giannini anche strumento di difesa e di attacco personale verso i leader e i partiti dell'antifascismo.

Mi sono servito dei miei mezzi, della mia arte ch'è fatta di maestria della battuta, ho risposto all'ingiuria con l'ingiuria [...] all'insinuazione malvagia con lo sberleffo, all'austera ipocrisia dei puri cialtroni con la caricatura e col pernacchio [;] a chi mi ha dato del fascista ho dato del fetente ${ }^{21}$.

Fra i suoi bersagli preferiti spiccava il Partito d'Azione, definito «il partito più ridicolo del bacino Centro-mediterraneo» ${ }^{22} \mathrm{e}$ «il più ridicolo dei partiti a est del meridiano di Greenwich» ${ }^{23}$. Così Piero Calamandrei diventava "Caccamandrei” o "Camaleontèi”, Luigi Salvatorelli il "filosofesso Servitorelli”, Ferruccio Parri, "Fessuccio Parri”. Gli azionisti si presentavano, in effetti, come i principali avversari del qualunquismo e del suo elogio del quieto vivere, in nome di un rinnovamento integrale delle strutture della società italiana e di una certa intransigenza morale. Gli attacchi che

19 ID., «Le Vespe», in UQ, III, 20, 15 maggio 1946.

${ }^{20}$ ID., «Le Vespe», in $U Q$, II, 15, 30 maggio 1945.

${ }^{21}$ Cit. in ZAMPAGLIONE, Gerardo, «Il Fronte dell'U.Q. si è costituito in partito politico durante i lavori del suo primo Congresso Nazionale - Guglielmo Giannini eletto Presidente del Fronte», in $U Q$, III, 8, 20 febbraio 1946.

${ }^{22}$ GIANNINI, Guglielmo, «Le Vespe», in UQ, II, 36, 24 ottobre 1945.

23 Ibidem. 
la stampa azionista ${ }^{24}$ riservava a «L'Uomo Qualunque» venivano però ribattuti colpo su colpo dalle vespe gianniniane. Gli stessi attacchi venivano rivolti ai comunisti, e soprattutto al ministro dell'agricoltura Fausto Gullo, ribattezzato "Fausto Grullo", promotore di una serie di interventi di riforma agraria: «Come l'acqua avversa il fuoco, come il molto aborre il poco, comunisti e proprietari son di solito avversari. Ma non è così citrullo il compagno Fausto Gullo, gran borghese, forte agrario, comunista e milionario»25. La questione giuliana e le accuse ai comunisti di fare il gioco dei titini venivano risolte consigliando a De Gasperi di «offrire Scoccimarro alla Jugoslavia, e tenerci il Friuli» ${ }^{26}$. Stesso trattamento riceveva Pietro Nenni, detto "capp'e provola". Osteggiato nonostante nei primi numeri del giornale gli fosse stato dedicato un articolo benevolo che lo ritraeva come avversario leale ${ }^{27}$, Nenni veniva paragonato a Mussolini per il suo accento: «Abbiamo udito romagnolità come "un vuomo", come "l'Itaglia", e se nel vecchio trombone ci fosse stato un po' di miglior metallo si sarebbe potuto, chiudendo gli occhi, illudersi d'ascoltare "l'altro" ${ }^{28}$. Ma soprattutto veniva definito «il foruncolo al culo della vita politica italiana»29.

Come ha osservato Angelo Panebianco30, accade spesso che, nella dinamica della competizione politica, gli attacchi più virulenti vengano riservati non agli esponenti del fronte opposto ma a quelli di partiti e movimenti contigui, ovviamente percepiti come concorrenti al favore dello stesso elettorato. Inizialmente, infatti, oggetto degli attacchi gianniniani furono il Partito Liberale e Benedetto Croce, considerato la «croce del partito liberale», responsabile di aver ostacolato la fusione fra liberali e qualunquisti.

Nessuno più di lui rassomiglia a quel tale che trovatosi inopinatamente in possesso d'un cannone lo utilizzò andando con esso a caccia di passeri. Il più grande, il più clamoroso, il più significativo insuccesso politico italiano è quello di Benedetto Croce ${ }^{31}$.

Con l'affermazione elettorale dell'Uomo Qualunque sia nelle elezioni politiche del 2 giugno 1946, che nelle amministrative dello stesso anno, il ruolo di partito competitore fu occupato dalla Democrazia Cristiana che, in prossimità delle elezioni, veniva descritta come il «partito-biscia, il partito anfibio, il partito equivoco per eccellenza; e, praticamente, non è nemmeno un partito perché è soltanto una clientela di

24 L'organo ufficiale del PdA era «L'Italia Libera» diretto da Leo Valiani.

25 GIANNINI, Guglielmo, «Le Vespe», in UQ, II, 20, 4 luglio 1945.

${ }^{26}$ ID., «Le Vespe», in $U Q$, II, 14, 23 maggio 1945.

${ }_{27}$ Cfr. ID., «L'Europeo Pietro Nenni», in UQ, I, 6, 7 febbraio 1945.

${ }^{28}$ ID., «Le Vespe», in UQ, II, 14, 23 maggio 1945.

29 ID., «Le Vespe», in UQ, IV, 11, 12 marzo 1947.

$3^{30}$ Cfr. PANEBIANCO, Angelo, Modelli di partito, Bologna, Il Mulino, 1982.

${ }^{31}$ GIANNINI, Guglielmo, «Croce del partito liberale», in UQ, III, 44, 30 ottobre 1946. 
professionisti della politica», composto di «demo-niente-cristiani»32. Giannini, infatti, dopo aver presentato fin dagli albori il suo come un movimento liberale, espressione del liberalismo più puro, «sfrondato delle sciocchezze dei nuovi e vecchi fregnoni del sedicente Partito Liberale Italiano e, principalmente, ripulito di quella criminosa e infruttifera cretinaggine che è l'anticlericalismo di maniera, il laicismo parolaio e inconcludente»33, andava rivendicando con sempre maggiore convinzione e insistenza la qualifica di partito cattolico. «Noi siamo democratici e cristiani, ma non tutte e due le cose insieme: non andiamo commerciando Iddio nei comizi, non diciamo alle contadine: "Se non voti per noi andrai all'Inferno". C’è una bella differenza!»34. Gli attacchi si andarono facendo sempre più intensi, man mano che la DC si venne profilando come il vero partito di riferimento per quell'elettorato cattolico e conservatore al quale pure l'UQ mirava. Una delle accuse più frequenti era proprio quella che la DC non tollerasse concorrenti, proprio «come quelle vecchie baldracche che insultano le compagne di lavoro»35. Fra le tante stoccate, la DC fu "punta" tramite una barzelletta (raccontata anche durante una seduta dell'Assemblea Costituente) sulla guerra civile spagnola che serviva a esemplificare i rapporti fra i due partiti, con il pappagallo nel ruolo dei qualunquisti.

La storiella narra l'avventura d'un pappagallo d'una nobile famiglia spagnola tremendamente monarchica, perseguitata dai repubblicani che in quel momento sono al potere. Ogni tanto irrompono nella vecchia casa hidalga nugoli di poliziotti repubblicani a compiere perquisizioni. Nella tema che il pappagallo possa ripetere qualche brano dei discorsi che solitamente si tengono in casa, i perseguitati monarchici lo nascondono durante le perquisizioni: e lo nascondono sotto le gonne della nonna, per essere più sicuri che il pappagallo se ne stia tranquillo. Per una volta, per due, il povero pappagallo resiste all'atmosfera mefitica del bizzarro nascondiglio, ma alla terza volta non ne può più, spacca con un colpo di becco la gonna della nonna hidalga, e si slancia all'aperto, nell'aria pura, gridando: «Prefiero la muerte, viva el Rey!»36.

Molto più avvezzo alla commedia teatrale che ai meccanismi della politica, Giannini utilizzò la satira anche per lavare, pubblicamente, i panni sporchi del suo partito. Alle prime contestazioni della sua posizione personale rispondeva con gli insulti a mezzo stampa.

$3^{2}$ ID., «Le Vespe», in UQ, II, 20, 15 maggio 1946.

33 ID., «Primo bilancio dell'Uomo Qualunque», in UQ, II, 21, 19 settembre 1945.

34 ID., «Le Vespe», in UQ, II, 20, 15 maggio 1946.

35 ID., «Le Vespe», in UQ, IV, 6, 5 febbraio 1947.

${ }^{36}$ ID., «Le Vespe», in UQ, IV, 41, 8 ottobre 1947. 
Ah ah ah! Ci dimenticavamo il meglio: c'è la curiosa situazione personale del dittatore del Fronte, e cioè di quel terribile tiranno che risponde, quando ne ha voglia, al nome di Giannini Guglielmo, di professione proprietario di alcune dozzine di corbelli molto resistenti, ma che, a furia di battervi sopra, si possono anche rompere. E ch'accadrebbe, ch'accadrebbe il giorno che quel dittatore e tiranno dicesse ai vari Manfredi, ai vari suoi scodinzolatori e famili: neh, voi me li avete rotti, trovatevi un altro dittatore e tiranno e andate definitivamente a farvi fottere?37

Ma fu soprattutto in occasione della crisi - poi irreversibile - che colpì il partito nel 1947 che ogni dissidente divenne bersaglio polemico delle vespe; questo destino spettò in particolare il deputato alla Costituente Enzo Selvaggi, "portatore di sventura" in quanto ritenuto responsabile della scomparsa prima della formazione monarchica del Partito Democratico Italiano e poi della crisi del Fronte dell'Uomo Qualunque. Giannini collocava i qualunquisti dissidenti nel "partito della forchetta", formato da «coloro che fanno i partiti per camparci sopra» ${ }^{8}$. Pure nel rispetto delle formalità parlamentari (il comportamento di Giannini e dei deputati qualunquisti in aula fu sempre impeccabile e non manifestò nessuno degli atteggiamenti classici del partito anti-sistema) ${ }^{39}$, Giannini trasportò nel linguaggio della politica i modelli comunicativi dello spettacolo. È allora abbastanza emblematico che, mostrando la sua riluttanza a entrare a far parte di un ministero quando De Gasperi glielo propose, rispose ironicamente di creare per lui il «Ministero delle Fregnacce», con la funzione di diffondere nel mondo la «giusta» immagine dell'Italia40.

Il "parlare chiaro", l'utilizzo del linguaggio popolare, non servivano solo a giustificare scelte politiche e a contrapporsi a moduli formali esistenti, ma svolgevano anche la funzione di esaltazione dell'opinione popolare, nel senso che a questa espressione ha dato Paul Corner, ovvero di dibattito spontaneo e non organizzato nella società civile ${ }^{41}$. La risata diventava quindi uno strumento d'opposizione ai regimi totalitari, i quali non «possono tollerare che si rida».

Essi sono seri come la morte, come la miseria di cui si servono come del loro migliore e peggiore strumento: hanno la necessità suprema di rompere i coglioni all'umanità, di tormentarla di farla penare sotto il peso della tristezza della vita

37 ID., «Le Vespe», in UQ, II, 45, 6 novembre 1946.

38 ID., «Le Vespe», in UQ, IV, 35, 27 agosto 1947.

39 Per il concetto di «partito anti-sistema» cfr. SARTORI, Giovanni, Teoria dei partiti e caso italiano, Milano, SugarCo, 1982.

40 GIANNINI, Guglielmo, «Il Ministero delle Fregnacce», in UQ, IV, 51, 17 dicembre 1947.

${ }^{41}$ Cfr. CORNER, Paul, Il consenso totalitario: Opinione pubblica e opinione popolare sotto fascismo, nazismo e comunismo, Roma-Bari, Laterza, 2012. 
d'ogni giorno, senza un'evasione, senza una schiarita di cielo, senza un sorriso, nell'infinita melanconia del mondo quadrato e balordo che il totalitarismo immagina, fatto per i vivi, mentre non è altro che un enorme sforzo per far rivivere i cimiteri ${ }^{42}$.

Sotto il fascismo non si rideva e così sembrava a Giannini essere nell'Italia antifascista: «nessuno ride, nessuno ha il coraggio di guardare il volto rugoso della realtà terribile facendole il pernacchio che merita. Nostra opinione personale è che il pernacchio può essere eroico in certi casi: lo è sempre quando è fatto sul volto della realtà»43. Era la celebrazione del cosiddetto jus murmurandi che Giannini riprendeva nella forma e nel contenuto per un'analisi non solo del presente ma anche del recente passato fascista. Le barzellette antifasciste, sotto questo punto di vista, servivano a testimoniare all'Italia moderata che il fascismo era stato un regime più nelle parole che nei fatti. Non è un caso che già nel 1944 veniva pubblicata a Roma una raccolta di barzellette antifasciste e, proprio su questa tema, scrive La Rovere:

L'arma del ridicolo e del grottesco era quella che meglio si prestava a dimostrare il fallimento della pretesa totalitaria del fascismo. Carlo Veneziani, un autore di operette cui era arrisa una certa notorietà negli anni venti, raccolse in un volume le barzellette, i motti di spirito e le facezie circolanti sul duce, sui gerarchi e sul fascismo durante il ventennio, con l'intento dichiarato di contribuire a comprendere l'atteggiamento degli italiani sotto la dittatura. Malgrado il clima di violenza e di intimidazione, il fiore della barzelletta, il suo diffondersi in tutti gli ambienti attraverso il passaparola, arrivando a lambire le alte sfere del potere, aveva assolto alla "funzione che milioni di oppositori non potevano compiere" corrodendo lentamente la credibilità del regime e dimostrando "quanto l'animo degli italiani fosse ostile allo stupido e bestiale dispotismo fascista" 44 .

Guglielmo Guasta, direttore del giornale satirico «Il Travaso», espulso dall'Albo dei giornalisti nel 1925 per "indegnità politica”, a causa degli sfottò che riservava al regime, e che scrisse anche su «L'Uomo Qualunque», racconta così i giorni seguenti alla caduta di Mussolini:

Il 25 luglio 1943 Mussolini è arrestato e il regime cade in polvere.

Di colpo, come se Dio Padre, infilando un braccio nello Stivale e afferratane dall'interno la punta, lo avesse voltato alla rovescia, la popolazione fu unanime

42 GIANNINI, Guglielmo, «Le Vespe», in UQ, IV, 40, 1 ottobre 1947.

43 Ibidem.

44 LA ROVERE, Luca, L'eredità del fascismo: Gli intellettuali, i giovani e la transizione al postfascismo 1943-1948, Torino, Bollati Boringhieri, 2008, p. 109. 
nell'applaudire all'evento: i distintivi (le spregiate "cimici") sparirono come al SimSala-Bim del prestigiatore: TUTTI ANTIFASCISTI e tutti DA SEMPRE.

I muri d'ogni strada e d'ogni piazza si coprirono di scritte ingiuriose e beffarde (una delle più caustiche si lesse a Firenze: "Mancia competente a chi riporterà un cane fascista!").

Qualcuno cercò di spiegare il fatto sorprendente osservando che quanti si erano iscritti al partito, o per interesse o per viltà, era naturale si precipitassero a cambiare bandiera, e quanti lo avevano fatto in buona fede, ritenendo il 28 ottobre 1922 una giornata liberatrice dal caos del dopoguerra, avevano avuto - e come! - il tempo di aprire gli occhi. Costoro salutarono con lo stesso entusiasmo la fine del regime dell'olio di ricino, del manganello, del confino e peggio, tale e quale a quelli che avevano rifiutato di indossare la camicia nera, riuscendo a mantenersi vergini di occhiello. Sia gli uni che gli altri si diedero a diffondere, compiaciutissimi, le brucianti battute e le barzellette sfottenti - nuove di zecca o vetuste e rielaborate che venivano ora scandite ad alta voce e stampate in corpo 10 dopo essere state, nel "deprecato ventennio", sussurate all'orecchio degli ascoltatori come si confida un segreto45.

D'altronde, come ha notato Francesco Perfetti, quello dello jus murmurandi è un mare magnum che

gli studiosi farebbero bene a solcare dal momento che le onde dell'ironia, della satira e, perché no?, anche dell'umorismo sboccato e goliardico finiscono sempre per fornire qualche, sia pur piccolo, contributo alla comprensione della temperie dell'epoca, degli stati d'animo cioè, degli umori, delle illusioni della gente comune oltre che dei difetti e delle manie di grandezza dei potenti 46 .

Se questo discorso vale per il ventennio non può che valere anche per gli anni della transizione alla democrazia; centrale era quindi nel discorso qualunquista la dissacrazione dei miti fondanti dell'antifascismo e della resistenza, dissacrazione che toccava l'apice nel ribaltamento dei canoni imperanti: il vero antifascismo non era stato quello degli oppositori dell'Aventino o dei fuorusciti o dei militanti comunisti, ma quello di tutti gli italiani che avevano sofferto sotto il fascismo e non «vissuto comodamente nei grandi alberghi stranieri ordendo facili congiurette al riparo dai pericoli»47. Già nel 1946, Michele Dipiero, scrivendo una prima Storia critica dei

45 GUASTA, Guglielmo, FERRI, Luciano, Jus murmurandi in camicia nera, Firenze, Le Lettere, 2009, p. 9.

46 PERFETTI, Francesco, Introduzione, in GUASTA, Guglielmo, FERRI, Luciano, Jus murmurandi in camicia nera, cit., p. IX.

47 GIANNINI, Guglielmo, «Il Muro di ghiaccio», in UQ, III, 52, 25 dicembre 1945. 
partiti italiani, vedeva nell'affermazione del qualunquismo un'impresa compiuta senza troppi sforzi, proprio perché capace di dare voce «allo jus murmurandi, di un popolo ancora una volta deluso» 48 .

Le crepe e le contraddizioni dell'esarchia, la insufficienza degli uomini, lo strapotere dei partiti, le preoccupazioni personalistiche e di parte sostituitesi ad una responsabile azione di governo, lo spirito di vendetta, lo sfogo delle ambizioni contrastate, il moralismo filisteo, la denuncia di una classe dirigente non diversa da quella che moralmente fu la classe dirigente fascista: tutti questo sono stati motivi della polemica dell'Uomo Qualunque49.

Giannini insomma dava forma ed espressione a un'opposizione inerte e silenziosa che, a suo giudizio, aveva lentamente abbattuto il fascismo che, infatti, «non è caduto alle 22,45 del 25 luglio 1943: a quel momento esso ha solo cessato di cadere» $5^{\circ}$. Negare, o sminuire, il ruolo svolto dai CLN e dalle personalità dell'antifascismo militante serviva quindi a esaltare la funzione dell'opinione popolare come vera e sincera, e quindi "non professionale", opposizione al regime.

Una storiella, di quelle che da sole fecero crollare il regime mussoliniano, nacque ad un tavolo di trattoria romana, alla quale sedeva un bel poeta nostro. C'era chi sosteneva che i membri della famiglia Ciano fossero tre, o due, e il poeta precisò ch'erano quattro: Ciano padre, Ciano figlio, Ciano zio e Ciano rotto li cojoni. Vale più questo "spirito borghese" che non una dozzina di cupi discorsi di oratori da comizio51.

L'utilizzo di questi canoni, pur condito da dichiarazioni di stima e rispetto per «l'antifascismo onesto» e le «vere vittime» del fascismo, minava le basi di legittimità del sistema ciellenistico.

Insomma si finisce sempre col tornare al motto fondamentale [su] cui si è costruito il formidabile edificio dell'U.Q. e cioè: non vogliamo essere più scocciati da nessuno! "Lei è fuoruscito? Tanto piacere, s'accomodi, e si pulisca i piedi prima d'entrare. Lei è vittimo [sic]? Bravo, qua la mano, ora le troveremo da sedere, prego, vada avanti e cerchi di dare il minor fastidio che può. Lei è antifascista? Fortunatissimo: ecco, caro collega, qui c'è tutta l'Italia, antifascista come lei, sappiamo tutti di che si tratta, stia con noi allegramente e smetta quella faccia da

48 DIPIERO, Michele, Storia Critica dei partiti italiani, Roma, AEI, 1946, p. 255.

49 Ibidem.

$5^{\circ}$ GIANNINI, Guglielmo, «Madonna Pace», in UQ, II, 2, 10 gennaio 1945.

${ }^{51}$ ID., «Le Vespe», in UQ, II, 14, 23 maggio 1945. 
fesso d'eccezione, perché qui, in un modo o nell'altro, abbiamo tutti sofferto". Ecco ciò che si deve dire e si dice: e basta ${ }^{2}$.

Il giornale puntava, e lo rivendicava ostentatamente, a identificarsi con un generico "popolo" italiano; Giannini non aspirava a essere soltanto un «annusatore dell'aria ammorbata che ristagna nei bassifondi della società, e che egli trattiene come in una spugna o in una carta assorbente», come ha scritto Silvio Lanaro53, si presentava invece come la voce autentica dell'uomo della strada. Il registro stilistico doveva allora essere quello popolare: «il grandissimo successo dell'Uomo Qualunque è dovuto al fatto che, all'origine, sono gli uomini qualunque che lo pensano: noi ci limitiamo a tradurre nella più semplice prosa il pensiero di migliaia di persone»54. A prescindere dalle reboanti dichiarazioni di Giannini, il successo de «L'Uomo Qualunque» fu, alla prova dei fatti, indiscutibile. Le ragioni di ciò vanno ricercate nella capacità di interpretare uno stato d'animo diffuso sì nell'Italia del dopoguerra, ma che era andato formandosi negli ultimi anni del regime e nel corso della guerra. Come avevano segnalato gli informatori fascisti, la novità del fascismo andava esaurendosi e la gente «era stanca di essere costretta a partecipare a tante attività fasciste, stanca delle (care e scomode) uniformi, stufa della prevaricazione e della disonestà dei capi locali e temeva per il proprio futuro»55. L'«Uomo Qualunque», come abbiamo visto, si poneva fin dal primo numero in continuità con questo tipo di stato d'animo (cioè di chi era «stufo di tutto») e manifestava la propria insofferenza nei confronti della transizione al nuovo sistema politico in nome di un non ben precisato «ritorno alla normalità». "Insomma il Sagittario [giornale] di Sulmona dice che stiamo con gli scontenti. E con chi dovremmo stare: con i contenti? Sagittario, non dire fesserie» 56 .

Il travolgente successo del giornale si tradusse anche nell'affermazione politica e personale di Guglielmo Giannini, che fu uno dei sei deputati eletti in tre circoscrizioni differenti (Napoli, Roma e Bari) e il candidato che raccolse il maggior numero di voti di preferenza, specialmente a Roma. Ne «L'Uomo Qualunque» Giannini riversò la sua esperienza di commediografo, affrontando la gravità del momento con il gusto del "pernacchio"; nelle parole di Sandro Setta, «Giannini ebbe successo proprio col suo travolgente "stil nuovo", ch'era un invito a sorridere, a dimenticare, almeno per un

\footnotetext{
$5^{2}$ ID., «Le Vespe», in UQ, III, 9, 27 febbraio 1946.

53 LANARO, Silvio, Storia dell'Italia repubblicana, Venezia, Marsilio, 1992, p. 129.

54 GIANNINI, Guglielmo, «Le Vespe», in UQ, II, 20, 4 luglio 1945.

55 CORNER, Paul, Il consenso totalitario, cit., p. 147.

${ }^{6}$ GIANNINI, Guglielmo, «Le Vespe», in UQ, II, 27, 22 agosto 1945.
} 
attimo, le lacrime e il sangue versato»57. Se è vero che l'indifferentismo ideologico, la tendenza al disimpegno sociale, la più generale diffidenza del cittadino nei confronti del potere politico costituiscono uno stato d'animo che nasce dallo strutturarsi dei sistemi democratici, Giannini trovò una formula vincente per dargli forma. La satira è legata a un contesto storico dal quale non la si può estrapolare senza rischiare di perderne di vista il significato. Si può sostenere per Giannini quanto Adolfo Chiesa ha sostenuto per un altro grande maestro della satira, Giovannino Guareschi, l'essere «un po' monarchici e un po' anarchici, certo reazionari ma capaci di dialogare con la gente, di far capire ai loro lettori, con decenni di anticipo, tanti malanni della politica, magagne della società, difetti delle istituzioni che solo tanto tempo dopo sarebbero venuti alla luce in tutta la loro evidenza»58. La satira fu quindi, come ampiamente rivendicato dal giornale, non solo un contenitore ma un contenuto in sé, un modo di differenziarsi, di offrire qualcosa di nuovo e diverso, di codificare un atteggiamento così come l'antipolitica è un modo di fare politica. Come sostiene Attilio Brilli, «la satira sancisce la supremazia dei significanti che a volte cancellano i significati»59. Probabilmente contribuì al successo di Giannini la sua riluttanza a indossare completamente i panni del politico, volendosi per l'appunto distinguere da quella categoria che lui aveva battezzato come "uomini politici professionali". Di fronte a un gruppo di comunisti e sardisti che lo contestavano durante un comizio a Cagliari, Giannini rispose portando la folla a cantare «Dove sta Zazà» ${ }^{60}$. Tutta la sua carriera politica fu segnata dall'uso di schemi e registri eccentrici rispetto ai canoni più rigidi della politica. Parte importante di questo stile era il fatto che non perdesse mai occasione di sottolineare la sua volontà di abbandonare la politica e tornare a tempo pieno al teatro, cosa che poi fece fino alla sua morte. Quando Montanelli lo incontrò, nei tardi anni '50, Giannini aveva perso anche quel poco di fiducia nella politica che la nascita del suo movimento poteva aver generato in lui.

Hai capito, Montane', com'è la storia dell'Uomo qualunque? Io m'ammalai, una fistola mi tenne a letto da Natale a Pasqua, e i vari assessori comunali di Rocca Priora ne approfittarono per litigarsi. L'uomo qualunque era diventato uomo politico e naturalmente si era discreditato agli occhi dei veri uomini qualunque, $i$ defraudati che vanno a prender l'acqua col secchio... Perché i defraudati, che

\footnotetext{
57 SETTA, Sandro, Il qualunquismo, in SETTA, Sandro, (a cura di), Gli Italiani contro gli uomini politici: il qualunquismo, Napoli, ESI, 2005, p. 123.

${ }^{8}$ CHIESA, Adolfo, La satira politica in Italia, Roma-Bari, Laterza, 1990, p. 6.

59 BRILLI, Attilio, Introduzione, in BRILLI, Attilio (a cura di), La Satira, cit., p. 44.

${ }^{60}$ Cfr. «Movimentato comizio politico a Cagliari : Giannini parla a una enorme folla», in Unione Sarda, LVIII, 286, 7 dicembre 1946.
} 
furono defraudati appunto dalla politica, la politica non la vogliono. La politica è... Prostituta! 61

La netta contrapposizione fra "uomo qualunque" e "uomo politico", l'incomunicabilità fra questi due mondi fu il cardine del discorso qualunquista. Non a caso, Giannini rivendicava quale maggior successo del suo movimento l'aver superato il "millenario" concetto di sottomissione al capo. «Perché mai la mia vita, quella dei miei figli, dei miei nipotini innocenti, debbono dipendere dalla buona riuscita delle trattative fra Eisenhower e Kruscev?» si chiedeva, in un memoriale pubblicato su «Oggi» nel 1959 e che possiamo definire come il suo testamento politico ${ }^{62}$. Come ha scritto Cofrancesco, «c'era in lui del "tersitismo" - il dileggio dei potenti - ma, soprattutto quella desacralizzazione della politica (di cui la guerra perduta indubbiamente, aveva posto le condizioni), che, comunque la si voglia giudicare è una conditio sine qua non di una democrazia liberale, laica, senza complessi». Alla "retorica impero", Giannini contrapponeva la commedia, distruggeva miti, introduceva nella politica la leggerezza.

È il punto d'incontro di Guglielmo Giannini con l'Italia giornalistica e letteraria che non si riconosce nel "vento del Nord" [...] e nei disegni di rigenerazione eticopolitica nutrita dai partiti del Cln (Pci, Psi, Pd'a.). I Guareschi, i Longanesi, i Flaiano, i Montanelli, i Giovanni Mosca ecc. ${ }^{63}$.

Citando Carnazzi, «la vera satira politica, irriverente e settaria, alimentata da un vigoroso odio di classe, si sviluppa con ampiezza nei momenti di più acceso scontro tra $i$ partiti e le fazioni» ${ }^{64}$. Le armi del ridicolo e dell'ironia trovano solitamente espressione nei periodi più critici della storia italiana: durante il secondo dopoguerra «riaffiorarono presto, favoriti dalle difficili circostanze storiche, il distacco delle vicende contingenti, l'agnosticismo, il culto esclusivo per la propria arte... cioè i mali, pronti a risorgere in ogni momento di crisi»65. Giannini codificò, anche senza inventare nuovi termini, ma riciclandone di vecchi, il linguaggio dell'antipolitica in Italia: linguaggio di estrema diffusione, utilizzato spesso anche da parte di uomini politici, fino alle vicende a noi contemporanee ${ }^{66}$.

${ }^{61}$ MONTANELLI, Indro, Gli Incontri, Milano, Rizzoli, 1967, pp. 77-78.

${ }^{62}$ GIANNINI, Guglielmo, «La grande avventura dell'Uomo Qualunque raccontata da Guglielmo Giannini», cit., p. 62.

63 COFRANCESCO, Dino, «Qualunquismo», in Dizionario del liberalismo italiano, Soveria Mannelli, Rubbettino, 2011, p. 845.

${ }^{64}$ CARNAZZI, Giulio, La satira politica nell'Italia del Novecento, Milano, Principato, 1975, p. 2

65 Ibidem, p. 165.

${ }^{66}$ Per alcune riflessioni sull'antipolitica cfr. IGNAZI, Piero, La fattoria degli italiani, Milano, Rizzoli, 2009 e CANTARANO, Giuseppe, L'Antipolitica: Viaggio nell'Italia del disincanto, Roma, Donzelli, 2000. 


\section{* L'autore}

Maurizio Cocco frequenta il secondo anno del Dottorato di ricerca in Storia Moderna e Contemporanea presso l'Università di Cagliari. Il suo campo di ricerca è l'antipolitica e il qualunquismo storico.

URL: < http://studistorici.com/progett/autori/\#Cocco >

\section{Per citare questo articolo:}

COCCO, Maurizio, «Le vespe qualunquiste e la satira politica», Diacronie. Studi di Storia Contemporanea: La satira fa storia. Eventi, pratiche, linguaggi, 29/10/2012,

URL:<http://www.studistorici.com/2012/10/29/cocco_numero_11/ >

\section{Diacronie Studi di Storia Contemporanea $\beta$ www.diacronie.it}

Risorsa digitale indipendente a carattere storiografico. Uscita trimestrale. redazione.diacronie@hotmail.it

Comitato di redazione: Marco Abram - Giampaolo Amodei - Jacopo Bassi - Luca Bufarale - Gianluca Canè - Alessandro Cattunar - Alice De Rensis - Barbara Galimberti - Deborah Paci - Fausto Pietrancosta - Matteo Tomasoni - Luca Zuccolo 